\title{
A Study on the Surface Hardness Obtained by Nitriding with a Plasma Focus Machine $^{\dagger}$
}

\author{
Thiam Oun TEH*, Arwinder SINGH, Jalil ALI, Chee An NG, \\ Xue Yinn NG, Jun Wen WONG, Sor Heoh SAW and Sing LEE
}

Inti International University, Persiaran Perdana BBN, Putra Nilai, Malaysia

('Corresponding author's e-mail: thiamoun.teh@newinti.edu.my)

Received: 20 December 2017, Revised: 22 December 2018, Accepted: 29 December 2018

\begin{abstract}
A dense plasma focus (DPF) machine, being a source of a powerful ion beam, can be useful in the modification of the surface properties of materials. Experimental investigations were carried out with a $3.3 \mathrm{~kJ}$ Mather-type DPF operating in nitrogen at a low chamber pressure with low carbon steel as the target material. It was found that the DPF ion beam implanted nitrogen onto the steel surface thereby causing a marked increase in surface hardness. The variation of pressure and target distances appear to affect the outcome of this nitriding technique with optimum hardness reached at the pressure setting of 1 Torr and at a target distance of $40 \mathrm{~mm}$ from the anode.
\end{abstract}

Keywords: Dense plasma focus, hardening, nitriding, wear, corrosion

\section{Introduction}

The introduction of nitrogen ions into the surface of steel has been known to affect the hardness of the steel as well as its resistance to wear and corrosion. The industrial use of gas nitriding began in the 1930 s as a thermochemical surface treatment to improve the wear and corrosion resistance as well as the fatigue endurance of steel parts. Gas nitriding was later followed by salt bath nitriding and then by a process called plasma nitriding [1], also popularly known as glow discharge nitriding. Although the methods may vary [2], the end result of nitriding is to introduce nitrides onto the surface of the material to enhance its surface properties. This study explores the method of nitriding by using the dense plasma focus machine. The dense plasma focus machine, unlike plasma glow discharge machines used in nitriding, does not produce a continuous glow from the passage of a constant electric current through a gas. Instead, it sends a large electrical current through a low pressure gas and causes it to ionize into a plasma which then pinches down on itself with an increase in plasma density and temperature. The dense plasma focus machine is a source of highly energetic ions from its pinch, thus making it suitable as an alternative means of implanting nitrogen ions onto the steel substrate. Al-Hawat obtained results that showed that the surface hardness increased with increasing shot number and decreased with increasing distance from the anode [3].

\footnotetext{
${ }^{\dagger}$ Presented at the $10^{\text {th }}$ International Conference on Plasma Science and Applications 2017: October $10^{\text {th }}-11^{\text {th }}, 2017$
} 
http://wjst.wu.ac.th

\section{Materials and methods}

The material investigated in this study is AISI 1020 cold rolled steel. The dimensions of the metal samples used in this study were $70 \mathrm{~mm}$ in length, $25 \mathrm{~mm}$ in width and $7 \mathrm{~mm}$ in thickness. In its raw form, the material was cut from a commercially available cold rolled steel bar. The process of cold rolling the bar to the desired thickness for commercial distribution imparts an increase in hardness to the metal through a process known as work hardening. This work hardened steel has uneven hardness due to uneven cold working on different sections of the steel bar [4]. The result was that a test sample cut from the steel bar displays a hardness that is not consistent along its surface. This is easily demonstrated by hardness testing at different spots on the test sample surface. To carry out a study on a sample with uneven hardness is most unlikely to yield meaningful results. Therefore, in order to remove the effects of work hardening on the test specimens, the specimens are required to undergo an annealing process.

Test specimens are placed in an annealing oven and heated to a temperature of 910 degrees Celsius for one hour and then slowly cooled in the oven. When the steel samples have been cooled down to room temperature, they undergo a polishing process to remove scratches caused by machining and handling as well as any oxide layers that have been formed. Following the polishing process, hardness testing of the samples is carried out to determine the post-annealed hardness.

The first test sample is placed in the dense plasma focus machine at a firing distance of $40 \mathrm{~mm}$ from the anode tip and then the vacuum chamber is evacuated and filled with nitrogen gas. The absolute nitrogen pressure is adjusted to 0.5 torr and then thirty shots are fired in the dense plasma focus machine. Following that, other test samples are used with different gas pressures and different distances from the anode tip. Each test sample has its set of nitrogen pressure and firing distance, but each sample is fired with 30 ion beam shots. The pressure settings are fixed at 0.5, 1.0, 1.5 and 2.0 Torr. The firing distances are set at 40,60,80, 100 and $120 \mathrm{~mm}$. Using 4 different pressures and 5 different firing distances, a total of 20 test samples are used (Figure 1).

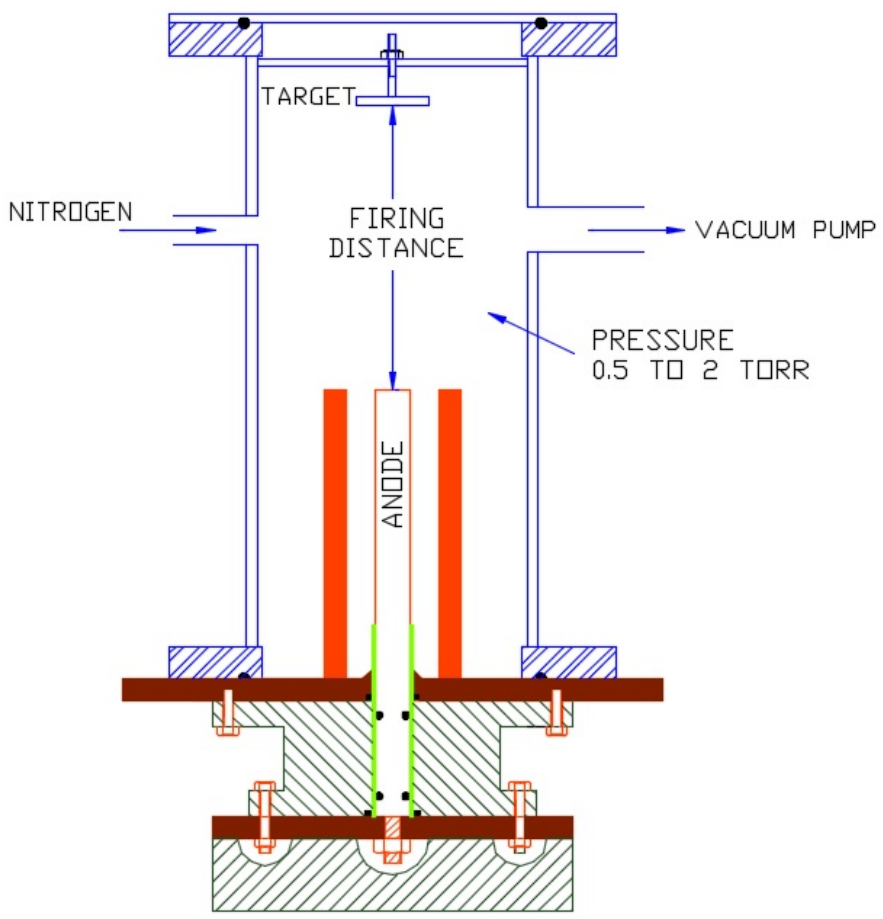

Figure 1 Dense plasma focus device. 
http://wjst.wu.ac.th

The nitrided test samples are then sent to the Micro Vickers hardness testing machine to have their hardness tested. The surface hardness is measured by using a $0.2 \mathrm{kgf}$ indentation load setting throughout the entire study. It is useful to set some kind of coordinate system on the sample in order to observe the variation of hardness improvement along the steel surface. The firing center is considered the zero point position for the purpose of convenience. This firing center has to be located visually by examining the ablation pattern on the surface. The hardness is then measured at every $1 \mathrm{~mm}$ distance from the center (or zero point) along the length of the sample.

\section{Results and discussion}

The firing process produces bands of discoloration on the test sample and it is easy to pinpoint the center of the firing effect by examining the test sample visually (see Figure 2).

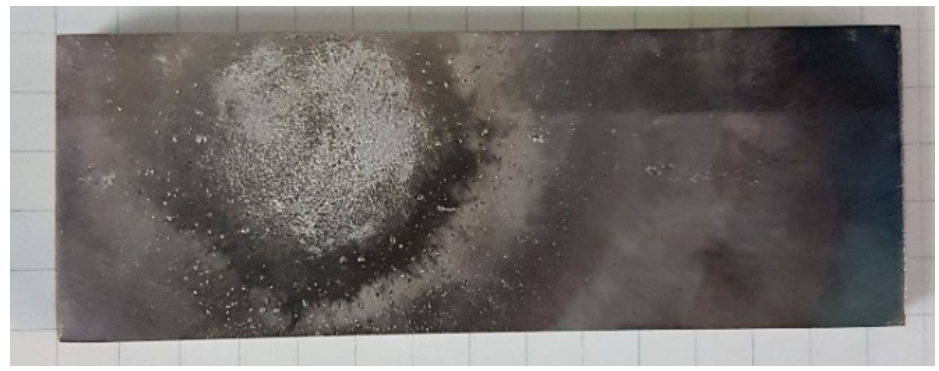

Figure 2 Center observed.

A series of ring patterns form around the center to allow one to easily pinpoint its location visually (Figure 3). The firing produced a small circular region, or sweet spot, that has a very rough appearance. Once out of the central region, the surface is smoother. A whitish pall is easily observed visually on the surface. Nitrided steel tends to have a "white layer" on the surface and therefore, the presence of a whitish pall on the steel samples would serve to imply that nitriding has taken place with a corresponding change in surface hardness.

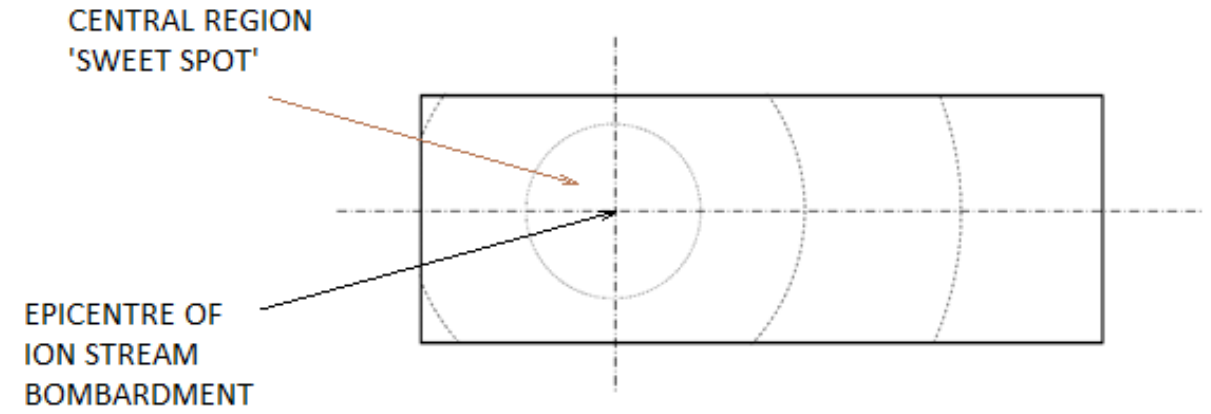

Figure 3 Central region and bands observed (drawn from Figure 2).

An energy dispersive X-ray (EDX) analysis done prior to firing (Table 1) indicated the absence of nitrogen in the sample. 
http://wjst.wu.ac.th

Table 1 EDX analysis before firing.

\begin{tabular}{lcc}
\hline Element & Weight percentage (\%) & Atomic percentage (\%) \\
\hline Carbon & 11.468 & 37.568 \\
Phosphorus & 0.041 & 0.052 \\
Sulfur & 0.050 & 0.061 \\
Manganese & 0.572 & 0.410 \\
Iron & 87.869 & 61.909 \\
\hline
\end{tabular}

After the sample has been treated in the plasma focus machine, an EDX analysis on the sample surface revealed the presence of nitrogen (Table 2).

Table 2 EDX analysis after firing (30 shots at 1 Torr $\mathrm{N}_{2}$ distance $40 \mathrm{~mm}$ ).

\begin{tabular}{lcc}
\hline Element & Weight percentage (\%) & Atomic percentage (\%) \\
\hline Carbon & 4.044 & 15.110 \\
Nitrogen & 3.212 & 10.290 \\
Phosphorus & 0.051 & 0.074 \\
Sulfur & 0.052 & 0.073 \\
Manganese & 0.502 & 0.410 \\
Iron & 92.139 & 74.042 \\
\hline
\end{tabular}

The presence of nitrogen serves to imply the presence of nitrides indicating that nitriding has taken place in the sample. Upon measuring the surface hardness, it was found that there was a variation in the hardness with the variation in firing distance and pressure parameters. The surface hardness of the steel samples is presented in Figure $\mathbf{4}$ where the variation in distance and pressure is clearly indicated. 

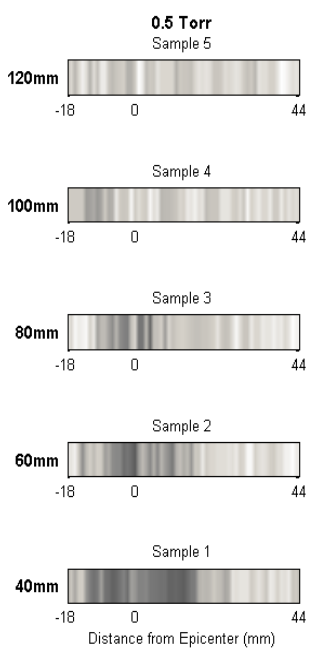
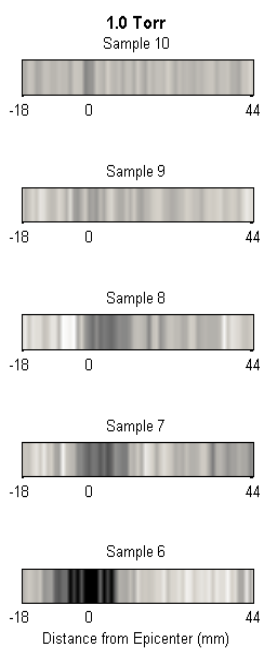
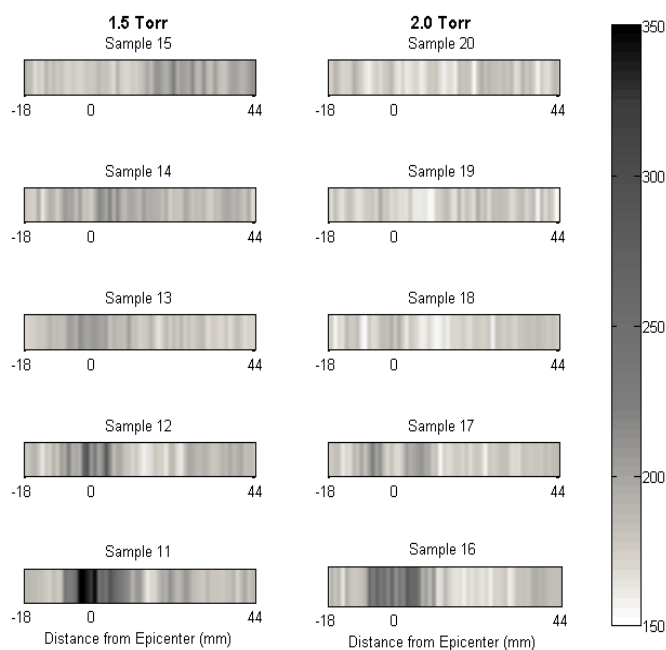

Figure 4 Micro Vickers hardness results in HV.

From Figure 4, the steel pieces that are placed at a firing distance of $40 \mathrm{~mm}$ above the anode provide the highest surface hardness at region close to the center of interaction which is assumed to be the center of the ion beam. Visual inspection revealed that the material experienced the greatest change in surface integrity in a small circular region around where it was most impinged on by the ion beam. This circular region is about 10 to $20 \mathrm{~mm}$ in diameter and also coincidentally has the highest surface hardness to be found on the sample.

The best results for hardness were obtained at the firing distance of $40 \mathrm{~mm}$ and at a pressure of 1.0 Torr of nitrogen gas. The center region (around point 0 in Figure 4) is the region which received the highest energy from the ion beam during the plasma shot. This energetic ion beam travelled to the steel target first to form a layer of iron nitride. It initiates the nitriding process on the surface of steel where the surface of steel will melt due to the high temperature, thus causing the steel to change in phase whereby ion energy is efficiently transferred for ion implantation. The melting and subsequent solidifying of the surface caused the initial smooth surface to take on a rough appearance at this center region. Once out of this central region, hardness tapers off rapidly.

The hardness results tend to be better with shorter travelling distances of the ion beam. The focusing efficiency of ion beam decreases (due to beam divergence) when it has to travel a longer distance to hit the target, hence causing a drop in the hardness attained. The variation in pressure used for the nitrogen gas indicates that pressure does have an influence on the results. The best result was obtained at 1.0 Torr. As the pressure is increased, the current pinches become weaker leading to a ion beam with less energy. This in turn will affect the results. At 0.5 Torr, the pinch is strongest as judged from the current dip, but the hardness obtained was not as good as that obtained at 1.0 Torr. Mean ion energies decrease with increasing gas pressure while the beam ion number increases with higher pressure [5-9]. At some point, both of these opposing factors will produce a state that will lead to optimum nitriding results. From this study, it appears to be at the pressure of 1.0 Torr.

\section{Conclusions}

Nitriding by using the dense plasma focus machine has been proven to be possible and the hardness that results depends on three parameters; 1) Pressure, 2) Distance of the sample from the anode and 3) Lateral position. From the pressure perspective, the pressure setting at 1 Torr of nitrogen yields the most favorable results. The distance of the sample from the anode appears to have an inverse relationship with 
http://wjst.wu.ac.th

the hardness and the further the distance of the sample from the anode, the less was the hardness obtained. It is observed that the hardness improvement tends to be concentrated in a small circular region. This central region is characterized by its rough appearance caused by localized melting induced by the energetic ion beam and the subsequent cooling of the surface and the accompanying solidification. The fact that the hardness tapers off rapidly from the small central region of ion bombardment implies that the dense plasma focus machine is more suitable for spot nitriding of a small area rather than the blanket nitriding of an entire steel part. Nevertheless, this method can still be useful in niche situations where only a small localized surface of a component is required to be hardened without affecting the rest of the component.

\section{References}

[1] M Lepicka and M Gradzka-Dahlke. Direct current and pulsed direct current plasma nitriding of ferrous materials: A critical review. Acta Mech. Automat. 2016; 10, 150-8.

[2] A Bernal. Investigation on Nitriding with Emphasis in Plasma Nitriding Process, Current Technology and Equipment. Materials Processing, Royal Institute of Technology, KTH, Stockholm, 2006.

[3] S Al-Hawat, M Soukieh, MA Kharoub and W Al-Sadat. Using Mather-type plasma focus device for surface modification of AISI304 steel. Vacuum 2010; 84, 907-12.

[4] F Sonmez and A Demir. Analytical relations between hardness and strain for cold formed parts. $J$. Mater. Proc. Tech. 2007; 186, 163-73.

[5] S Lee and SH Saw. Plasma focus ion beam fluence and flux: Scaling with stored energy. Phys. Plasmas 2012; 19, 112703.

[6] S Lee and SH Saw. Plasma focus ion beam fluence and flux: For various gases. Phys. Plasmas 2013; 20, 062702 .

[7] M Akel, SA Salo, SH Saw and S Lee. Properties of ion beams generated by nitrogen plasma focus, J. Fusion Energ. 2014; 33, 189-97.

[8] RS Rawat. Dense Plasma Focus -High-Energy-Density Pulsed Plasma Device Based Novel Facility for Controlled Material Processing and Synthesis. Plasma Science and Technology for Emerging Economies, AAAPT Experience, 2017, p. 39-112.

[9] S Lee and SH Saw. The Plasma Focus: Numerical Experiments, Insights and Applications. Plasma Science and Technology for Emerging Economies, AAAPT Experience, 2017, p. 113-232. 